\title{
Unforced Errors bei Studien- und Abschlussarbeiten
}

\author{
Hinweise zur Endredaktion
}

Vor der Abgabe einer Studien- oder Abschlussarbeit ist dringend eine sorgfältige Überarbeitung in Form einer Endredaktion vorzunehmen, um eine gute Bewertung der Arbeit nicht zu gefährden. Dies ist einfach; denn das Vorgehen ist schlicht und wenig aufwändig. Daher wäre es besonders ärgerlich, einfache Fehler nicht zu beheben und dafür Abzüge bei der Bewertung der Arbeit hinzunehmen. Für eine Endredaktion wird hiermit eine Anleitung vorgelegt.

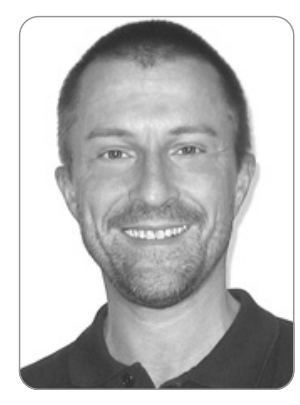

Prof. Dr. Georg Disterer

ist Professor an der Hochschule Hannover. Bevorzugte Forschungsschwerpunkte: Informationsmanagement, IT-Service Management, IT-Compliance und Projektmanagement.

Stichwörter: Studienarbeit, Abschlussarbeit, vermeidbare Fehler, Endredaktion, Checkliste

\section{Vermeidbare Fehler}

Beim Anfertigen einer Studien- oder Abschlussarbeit ist nach Abschluss aller inhaltlichen Arbeiten sowie nach der Anfertigung aller Texte, Abbildungen und Tabellen - als zusätzlicher Arbeitsschritt - eine Endredaktion vorzunehmen, bei der einfach zu vermeidende Fehler korrigiert werden. Im Sport steht der Ausdruck „unforced error" für Fehler, die im sportlichen Wettstreit nicht vom Gegner ausgelöst werden, sondern selbstverschuldet - und damit besonders ärgerlich - sind. Der Ausdruck "unforced error" soll hier für Fehler in Studien- oder Abschlussarbeiten stehen, die leicht vermeidbar sind und nicht vom Thema oder von der Aufgabenstellung einer Arbeit verursacht werden. Eine Vermeidung dieser Fehler ist relativ einfach; denn das Vorgehen ist schlicht und wenig aufwändig.

Unforced Errors sind leicht erkennbar und behebbar und daher - wenn sie trotzdem gemacht werden - besonders är- gerlich für die Autoren/innen. Hier ist eine Reihe besonders häufiger "unforced errors" angegeben, die als Checkliste zur Endredaktion genutzt werden kann. Weitere Hinweise und Beispiele sind zu finden bei Disterer (2019).

\section{Sorgfalt}

Bei Studien- oder Abschlussarbeiten ist größtmögliche Sorgfalt aufzuwenden, da Sorgfaltsfehler nicht nur dem Äußeren der Arbeiten schaden, sondern weil mangelnde Sorgfalt leicht auf mangelnde Korrektheit beim Denken schließen lässt. Sorgfalt mit dem Äußeren von Arbeiten wird manchmal - fälschlich - umgangssprachlich als "Formalkram“ abgetan, nach dem Motto: „Es kommt doch auf den Inhalt an!“. Dies ist jedoch eine Ausrede. Kaum jemand ist in der Lage, einen längeren Text ohne mehrmalige Korrekturrunden fehlerfrei anzufertigen. Daher sind diese Korrekturrunden einzuplanen und auch tatsächlich durchzuführen. Empfehlenswert ist, letzte Textkorrekturen nicht am gleichen Tag vorzunehmen, an dem ein Text entstanden ist. Vielmehr sollte damit ein oder zwei Tage gewartet werden, bis diese Aufgabe mit ausgeruhten Augen und geschärftem Blick erledigt wird. Andernfalls - zeigt die Erfahrung - werden Fehler leicht übersehen.

Selbstverständlich sind in Studien- oder Abschlussarbeiten die Regeln zur Orthografie, Grammatik und Interpunktion der deutschen Sprache zu beachten. Besonders ärgerlich sind Schreibfehler, die durch das Verwenden falscher Buchstaben und Buchstabenfolgen entstehen. Schon kleine Fehler erzeugen Missverständnisse, sogar schon bei einem einzigen Buchstaben oder Zeichen, wie 
z. B. bei "ratlos" und "rastlos", "stützen“ und "stürzen”, "Plage" und "Klage", bei der Groß-/Kleinschreibung bei "Die Spinnen ..." und "Die spinnen ..." sowie "Der gefangene Floh ..." und "Der Gefangene floh ..." oder bei Leerzeichen zum Trennen bei "zusammenbrechen“ und „zusammen brechen“. Auch kleine Fehler in der Zeichensetzung können gravierende Wirkung entfalten, wenn z. B. "... komm wir essen 0pa ..." auf manchen älteren Menschen lebensbedrohlich wirkt, während das Komma an der richtigen - in diesem Fall dritten - Stelle die Aussage zu einer freundlichen Einladung zur gemeinsamen Speise werden lässt.

Ein nützliches Mittel gegen Schreibfehler bieten Funktionen zur Rechtschreibkorrektur der gängigen Textverarbeitungsprogramme. Diese Funktionen markieren automatisch Wörter, die nicht in einem hinterlegten Wörterbuch enthalten sind. Die Wörter sind dann manuell zu prüfen, ob sie tatsächlich falsch geschrieben und daher zu korrigieren sind, oder ob es Wörter sind - zum Beispiel Fachausdrücke -, die nicht im Wörterbuch enthalten sind. Bekanntlich sind diese Funktionen zur Rechtschreibkorrektur nicht fehlerfrei, erfahrungsgemäß sind diese Fehler aber selten, zumindest seltener als Schreibfehler.

Besondere Aufmerksamkeit ist auf die Vollständigkeit aller Sätze zu legen. Textverarbeitungsprogramme erleichtern Einfügungen und Umstellungen von Texten erheblich, dabei geht aber leicht der Blick für Satzstrukturen oder auf Anschlüsse zu vorgehenden oder nachfolgenden Sätzen verloren. Eine einfache und wenig aufwändige Methode zum Auffinden dieser Fehler ist es, die Arbeit einmal betont langsam und laut (!) vorzulesen. Selbst wenn die Autoren/ innen dabei die einzigen Zuhörer/innen sind, wird deren Aufmerksamkeit durch den Moduswechsel von schnellem und stillem Lesen zum langsamen und lauten Lesen meist so geschärft, dass fehlerhafte Sätze und Unebenheiten im Text erkannt werden. Gewiss funktioniert diese Methode noch besser, wenn ein wohlgesonnener Mitmensch aufmerksam zuhört und auf Unstimmigkeiten hinweist.

Um ein möglichst ansprechendes Textbild von Arbeiten zu erzeugen, ist die Trennung von längeren Worten am rechten Rand der Texte vorzunehmen. Andernfalls entsteht ein sehr unruhiger und unansehnlicher rechter Rand - "Flatterrand“ - oder im Blocksatz relativ große Abstände zwischen den Worten. Hilfe leisten dabei gängige Textverarbeitungsprogramme mit Funktionen zur Silbentrennung, die automatisch Wörter an geeigneten Stellen trennen.

\section{Ausdruck und Sprache}

Die passende Ausdrucksweise in einer Studien- oder Abschlussarbeit ist gekennzeichnet durch Merkmale wie sachlich, klar, eindeutig, prägnant, nachvollziehbar. Nach- drücklich zu vermeiden ist eine Ausdrucksweise, die journalistisch, nichtssagend, weitschweifig, missverständlich oder floskelhaft zu nennen wäre. Umgangssprachliche Ausdrücke und Formulierungen sind zu meiden, da sie der angestrebten Seriosität einer Arbeit schaden.

Viele Wörter der deutschen Sprache können in einem Gespräch zwischen Freunden (vielleicht) genutzt werden, sind jedoch in einer schriftlichen Ausarbeitung zu einem Fachthema deplatziert. In diesem Sinne: Jede/r soll gerne einen eigenen Stil entwickeln, im Ergebnis muss jedoch ein Text entstehen, dessen Sprache angemessen für die Kommunikationsform und das Thema ist. Daher verbietet sich eine große Anzahl von Vokabeln und Ausdrücken, wie z. B. irgendwo, -wie, -was, -ein, in etwa, mehr oder weniger, quasi, an und für sich, ab und zu, hübsch, leider, ziemlich. Zur Verstärkung von Begriffen sind ungeeignet: riesig, phänomenal, immens, sagenhaft, himmelschreiend, toll, krass, hip, cool. $\mathrm{Zu}$ vermeiden sind nahezu alle Endungen mit -mäßig wie zahlenmäßig, ergebnismäßig, renditemäßig. Bildhafte Sprache und Redewendungen, die einen mündlichen Beitrag manchmal anschaulich und fesselnd werden lassen, sind in schriftlicher Form meist albern und unpassend, wie z. B. „Bauchlandung machen“, "auf der Hand liegen“, "danebengehen“, "Zahn der Zeit", "dünn ausfallen“, „breite Masse“, „Tal der Tränen“. Redewendungen wie "Gedanken machen“, "nie und nimmer", "voll und ganz" oder „immer und ewig“ sind in Studien- oder Abschlussarbeiten „voll daneben“. Ironische Bemerkungen und Witze sind fehl am Platz.

Gewarnt sei vor Sprachbildern, die bei den Lesern/innen falsche oder keine Assoziationen auslösen. Leser/innen erwarten keine sprachlichen Abenteuer, sondern klare und präzise Darlegungen. Bilder und Vergleiche können dabei wichtige Hilfe leisten, aber nicht blumige Ausdrücke um des Effekts willen; journalistische Ausdrücke sind immer unangemessen. Einige Beispiele dafür, dass effektvolle Formulierungen nur Aufsehen erregen, Leser/innen jedoch bestenfalls ahnen werden, was gemeint ist: „.... Benutzerfreundlichkeit muss großgeschrieben werden “, ".... das Innovationspotential ist noch lange nicht am Ende“, „.... hinter jedem Datensatz steht potenziell ein Mensch“. Mit derartiger Effekthascherei vertrauen Autoren/innen der Fantasie und Gutmütigkeit der Leser/innen, schon "das Richtige" aus den Formulierungen herauszulesen.

Zudem leidet der Anspruch der Seriosität einer Studienoder Abschlussarbeit deutlich, wenn das Sprachniveau an reißerische Darstellungen der Boulevardpresse erinnert, wie z. B. ".... Mannigfaltigkeit der Optionen“, „... die Konkurrenz ausstechen“ ${ }^{\prime}$, ... die Ärmel hochkrempeln“ ${ }^{\prime}, \ldots .$. das Budget sprengen“.

Einfache und klare Begriffe benötigen keine scheinbare Aufwertung durch sinnleere Verzierungen; Beispiele: Be- 
grifflichkeit (statt Begriff), formalistisch (statt formal), Regelung (statt Regel), überprüfen oder nachprüfen (statt prüfen), Zweckbestimmung (statt Zweck oder Bestimmung), Zielvorstellung (statt Ziel oder Vorstellung).

Sprachliche Verwicklungen und Verschlingungen "gereichen nur in geringem Maße der Verständigung und leisten suboptimalen Beitrag zur Rezeption und zur intellektuellen Durchdringung von Texten“; Beispiele: „unter Beweis stellen“ statt "beweisen“, ,zur Anwendung bringen“ statt „anwenden“. Auch eine Sprache, die mit der Attitüde eines erhobenen Zeigefingers daherkommt, weist auf eine unpassende Zuwendung von Autoren/innen zu Lesern/innen, z. B. "... man muss immer beachten ...", , ,... dabei darf niemals außer Acht gelassen werden ...".

Nichtssagende Floskeln und Satzhülsen wie "betrachtet man ..., so fällt auf, ..." sind zu vermeiden, da sie die Leser/innen unnötig langweilen; weitere Beispiele: „... was man mit Sicherheit sagen kann ...", „,... es muss festgestellt werden ...", „... dazu sei noch erwähnt ...“. Hauptsachen sollten im Hauptsatz formuliert werden, nicht im Nebensatz; z. B. statt "Jedoch ist darauf hinzuweisen, dass dies schwierig ist" besser: "Dies ist jedoch schwierig", statt „Es lässt sich feststellen, dass der wirtschaftliche Zusammenhang deutlich ist" besser "Der wirtschaftliche Zusammenhang ist deutlich".

In der deutschen Sprache können relativ einfach zusammengesetzte Begriffe gebildet werden, jedoch sollten Wortzusammensetzungen sinnvoll und verständlich sein. Jedenfalls nicht sinnvoll sind alle Zusammensetzungen, die eine Doppelung des Wortsinns enthalten, wie z. B. in "Grundprinzip": Schon das Wort "Prinzip" allein birgt den Charakter des Grundsätzlichen, "Grundprinzip" ist eine unnötige Doppelung. Ähnliches gilt für: Grundkonzept, Grundtenor, Perspektivstudie, Rückantwort, Frühpionier, Zukunftsprognose, Teilkomponente. Auch muss bei Zusammensetzungen klarwerden, was gemeint ist. So sollten Worte nicht allzu wahllos zusammengesetzt werden, da unklare, unscharfe oder schlicht hässliche Ausdrücke die Folge sind. Insbesondere die Mischung verschiedener Sprachen bei der Bildung zusammengesetzter Worte ist zu vermeiden; dafür nur wenige Beispiele: Unterhaltungselektroniksektor, Wissensetage, Softwarebusiness, Einzelteilkonglomerat.

\section{Präziser Einsatz von Fachbegriffen}

Häufig werden Begriffe der Fachsprache durcheinandergewürfelt oder schlicht falsch eingesetzt. Beispiel: In der Umgangssprache mag es gleich sein, vom "Internet" oder vom „WWW“ zu sprechen. Für Studien- oder Abschlussarbeiten jedoch gilt: "Internet“ und „WWW" bezeichnen zwei verschiedene Objekte, die zu unterscheiden lohnt. Andernfalls lässt unpräziser Umgang mit der Fachsprache schnell auf unpräzise Kenntnisse im Fach schließen.

Ein ähnliches Beispiel: Der Begriff „Firma“ steht für den Namen, unter dem Kaufleute oder Handelsgesellschaften Geschäfte betreiben. Nur in der Umgangssprache steht der Begriff "Firma” für „Unternehmen”. Damit verwenden Formulierungen wie „... das System wird in der Firma eingesetzt ..." oder „... der Umsatz der Firma beträgt ..." den Begriff fachlich falsch; jedes Mal ist „Firma” durch „Unternehmen" zu ersetzen.

Fachbegriffe sind konsistent, also durchgängig einheitlich einzusetzen und nicht wechselnd. Am Beispiel: In manchen Zusammenhängen wird zwischen den Fachbegriffen "Anwendungsprogramm" und "Anwendungssystem" kaum unterschieden werden können. Dann ist eines der beiden Begriffe zu wählen und dann durchgängig zu nutzen. Sonst wird bei wechselndem Einsatz der Begriffe bei Leser/in der Verdacht geschürt, dass es sich eben doch um verschiedene Dinge handelt. Also sollten die Begriffe nicht abwechselnd eingesetzt werden, etwa unter der irrigen Annahme, dass Leser/in nicht gelangweilt werden solle. Der Unterhaltungswert des Lesens einer Studien- oder Abschlussarbeit ist zweitrangig, der präzise Einsatz von Fachbegriffen vorrangig.

\section{Beachtung formaler Richtlinien}

Die äußere Form einer Studien- oder Abschlussarbeit ist an vielen Details sichtbar, z. B. Seitenformat, Seitenränder, Schriftart/-größe, Zeilenabstand/-ausrichtung, Aussehen des Deckblatts. In aller Regel gibt es für diese formalen Details Vorgaben von den Fachbereichen oder Hochschulen. Autoren/innen von Studien- oder Abschlussarbeiten sollten sich dringend diese offiziellen Vorgaben beschaffen (Holschuld) und diese dann kompromisslos anwenden. Dies hat durchaus auch Vorteile: Mit Hilfe der Vorgaben brauchen Autoren/innen keine eigenen Überlegungen zu vielen formalen Details anzustellen, da andere dies schon für sie erledigt haben. Während der Endredaktion einer Arbeit ist genau zu überprüfen, ob alle Vorgaben tatsächlich vollständig beachtet sind.

Insbesondere die Angabe verwendeter Fachliteratur und die Form von Zitaten unterliegt formalen Richtlinien. Die Verwendung von Fachliteratur ist bei der Erstellung von Studien- oder Abschlussarbeiten notwendig; Ausnahmen von dieser Regel sind nicht bekannt. Die Verwendung der Fachliteratur ist zu dokumentieren; diese Vorschrift hat den Charakter eines Gesetzes. Insoweit ist die Notwendigkeit der Verwendung von Fachliteratur und des Nachweises der Verwendung klar geregelt.

Zur ordnungsgemäßen Dokumentation der Verwendung von Fachliteratur dienen in aller Regel sog. Zitierrichtlini- 
en der Fachbereiche oder Hochschulen. Autoren/innen von Studien- oder Abschlussarbeiten sollten sich dringend diese Zitierrichtlinien beschaffen (Holschuld) und dann diese Regeln in Fußnoten und Literaturverzeichnissen kompromisslos anwenden. Nicht zulässig ist beispielsweise, eine „private“ Auswahl der Regeln zu treffen und nur diese anzuwenden, die restlichen Regeln jedoch unbeachtet zu lassen. Im Übrigen bekommen Zitierrichtlinien durch die Unsicherheiten im Umgang mit ihnen und die daher immer wieder aufkeimenden Diskussionen eine Bedeutung, die ihnen eigentlich nicht zusteht. Die Richtlinien stellen lediglich Formvorschriften und Konventionen dar, die aus vielen guten Gründen $\mathrm{zu}$ beachten sind. Sie haben damit ähnliche Bedeutung wie viele wohl begründete Formvorschriften und Konventionen im täglichen Leben.

Die Vorgaben in Zitierrichtlinien sind meist klar und einfach, deren Einhaltung ist keineswegs kompliziert. Lediglich sind Präzision und Sorgfalt bei Zitaten und Literaturangaben notwendig. Zum Beleg dieser These „Vorgaben sind nicht kompliziert, Einhaltung erfordert nur Präzision und Sorgfalt" sei angeführt: Bei Vorliegen des vollständigen Datenmaterials zu den Literaturquellen ist die ordnungsgemäße Erstellung eines Literaturverzeichnisses mit jeder höheren Programmiersprache einfach und auf weniger als zwei Seiten automatisierbar. Daraus ist zu schließen, dass dies nicht "kompliziert“ ist, sondern lediglich die präzise und sorgfältige Einhaltung einer überschaubaren Anzahl von Vorgaben erfordert.

\section{Einsatz von Abbildungen und Tabellen}

Abbildungen und Tabellen sollen in Studien- und Abschlussarbeiten eingesetzt werden, wenn sie zum Verständnis oder zur Übersicht beitragen. Sie dienen nicht als Schmuck oder zur Unterhaltung der Leser/innen, sondern der Übersicht, der Veranschaulichung von Abhängigkeiten oder zur Darstellung quantitativer Ergebnisse. Allerdings gilt für Abbildungen und Tabellen: Sie können textliche Aussagen nicht ersetzen, sondern lediglich ergänzen und unterstützen.

Eine Abbildung erspart also nicht die textliche Formulierung einer Aussage, sondern gibt die Chance, den Lesern/ innen zusätzlich zum Text mit einer Abbildung Verständnis zu vermitteln. Dafür ist den Lesern/innen im Text genau und ausdrücklich anzuzeigen, an welcher Stelle und wofür eine Abbildung zum Verständnis beitragen soll. Übliche Formulierungen hierzu lauten etwa "siehe dafür $A b b$. $7^{\prime \prime}$ oder "Abb. 7 verdeutlicht, dass ...". Nicht nur unhöflich, sondern unzulässig ist es, eine Abbildung irgendwo in einen Text einzustreuen und es den Lesern/innen zu überlassen, den Zusammenhang zwischen Text und Abbildung auszuforschen.
Abbildungen können auch Aufmerksamkeit erregen und auf wichtige Aspekte oder Zusammenhänge lenken. Zudem geben sie den Augen der Leser/innen Gelegenheit, bei langen textlichen Darstellungen zwischendurch ein wenig zu erholen. Abbildungen können so "Oasen in der Bleiwüste" sein. Dabei sollte der dekorative Effekt nicht überwiegen, daher sind spezielle Effekte (3D, Schatten ...) sparsam zu verwenden, da sie ohne Semantik nur Make-up sind.

Tabellen werden eingesetzt, um zum Verständnis oder zur Übersicht Daten oder Texte verdichtet darzustellen. Insofern können Tabellen einen Text ergänzen. Wie bei Abbildungen ist den Lesern/innen im Text genau anzuzeigen, an welcher Stelle und wofür eine Tabelle zum Verständnis beitragen soll. Übliche Formulierungen hierzu lauten etwa "siehe Tab. 2" oder "Tab. 2 zeigt einige Beispiele dafür". Die Nutzung von Tabellen bietet einen Vorteil: Da sie mit einer Verdichtung der Übersicht dienen sollen, ist es zulässig, die Schriftgröße und den Zeilenabstand in Tabellen (etwas) kleiner zu wählen als im Text der Arbeit. Auch ist die Bildung vollständiger Sätze nicht immer notwendig, Worte oder Satzfragmente sind in einer Tabelle ausreichend.

\section{Aussagen ohne Beleg, Annahmen ohne Begründung}

Immer wieder sind in Studien- und Abschlussarbeiten Aussagen zu lesen, die weder begründet noch belegt sind. Beispiele dafür sind: Die Durchführung einer Bundestagswahl kostet ca. 60 Mio. Euro; oder: Seit Ende des 19. Jahrhunderts können Erfindungen patentiert werden. Das Muster ist deutlich: Aussagen, die Kenntnisse über ein Faktum oder einen Zusammenhang vorgeben, sind zwingend $\mathbf{z u}$ belegen oder zu begründen. Dies hat zumindest durch eine logische und nachvollziehbare Argumentation zu erfolgen, möglichst sind Quellen aus der Fachliteratur anzuführen, die eine Bestätigung für das angegebene Faktum liefern. Andernfalls ist der Reifegrad derartiger Aussagen mit denen von Behauptungen gleichzusetzen.

Insbesondere sind offensichtliche Übernahmen aus anderen Quellen mit Quellenangaben zu belegen. So weisen Formulierungen wie "Die Fachliteratur unterscheidet in diesen Fällen ...", "Der Experte Manfred Maier argumentiert, dass ..." oder „Der Umsatz des Unternehmens beträgt ..." unmittelbar und deutlich darauf hin, dass hier Wissen aus anderen Quellen wiedergegeben wird. Diese Quellen sind gemäß der jeweiligen Formvorschrift exakt anzugeben.

In vielen Studien- oder Abschlussarbeiten ist die Nutzung von Daten aus fremden Quellen notwendig. Die vollständige Angabe der Datenquelle ist dabei selbstverständlich. Dabei ist bei Nutzung externer Statistiken die Herkunft der Daten zu beachten. So sind etwa Absatzprognosen mit Vorsicht zu verwenden, wenn sie von Anbietern stammen. Of- 


\section{Logistik im Überblick.}

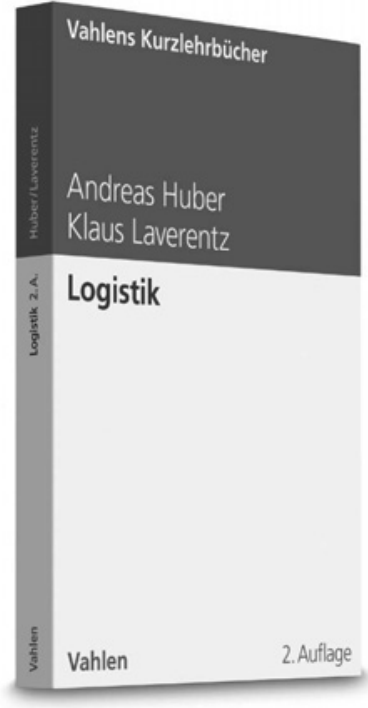

fensichtlich sind Anbieter nicht unvoreingenommen, sodass deren Zahlenangaben kritisch zu hinterfragen und wenn möglich - durch Angaben aus anderen Quellen zu stützen sind.

\section{Fazit}

Die Beachtung der hier aufgeführten Regeln und die Anwendung der empfohlenen Vorgehensweisen zur Vermeidung leicht vermeidbarer Fehler wandeln eine "schlechte" Studien- oder Abschlussarbeit zwar nicht in eine "gute". Jedoch können damit viele leichte Fehler erkannt und korrigiert werden, die sonst $\mathrm{zu}$ Abwertungen in der Beurteilung der Arbeiten führen.

\section{Literatur}

Disterer, G., Studien- und Abschlussarbeiten schreiben - Seminar-, Bachelor- und Masterarbeiten in den Wirtschaftswissenschaften, 8. Aufl., Berlin, Heidelberg 2019.

Huber/Laverentz

Logistik

2. Auflage. 2019. XIV, 254 Seiten. Gebunden $€ 19,80$ ISBN 978-3-8006-5889-3

\section{Perfekter Einstieg}

Das Lehrbuch behandelt den klassischen Lehrstoff der Logistik und berücksichtigt gleichzeitig auch aktuelle Entwicklungen. Ziel des Buches ist es, den theoriebezogenen und praxisrelevanten Stoff der Logistik zu verzahnen.

\section{Umfassend}

Neben den klassischen Funktionen der Beschaffungslogistik, Produktionslogistik und Distributionslogistik stellt das Buch ein modernes Dienstleistungsverständnis von Logistik dar. Integriert werden insbesondere Supply Chain Management, logistische Kommunikations- und Informationssysteme, Qualitätsmanagement sowie Lager- und Transportmanagement.

\section{Vahlen}

